\title{
INFLUÊNCIA DO SISTEMA DE CONDUÇÃO NA PRODUÇÃO E NA QUALIDADE DOS FRUTOS DAS VIDEIRAS 'FOLHA DE FIGO' E 'NIAGARA ROSADA' EM CALDAS, MG' ${ }^{1}$
}

\author{
Influence of the training system in the yield and in the quality of vine fruits 'Folha de Figo' \\ and 'Niagara Rosada' in Caldas, MG
}

\author{
Paulo Márcio Norberto ${ }^{2}$, Murillo de Albuquerque Regina ${ }^{3}$, Nilton Nagib Jorge Chalfun ${ }^{4}$, \\ Angela Maria Soares ${ }^{5}$, Vanessa Brito Fernandes ${ }^{6}$
}

\begin{abstract}
RESUMO
O presente trabalho foi desenvolvido na Estação de Viticultura e Enologia da EPAMIG em Caldas-MG, e objetivou avaliar a influência do sistema de condução na produção e na qualidade dos frutos das videiras 'Folha de Figo' e 'Niagara Rosada' durante as safras 2003, 2004 e 2005. Como cultivares copa utilizou-se 'Niagara Rosada' e 'Folha de Figo', enxertadas no porta-enxerto '420-A'. $\mathrm{O}$ delineamento experimental foi inteiramente casualizado, em fatorial $4 \times 2$. Os sistemas de condução adotados foram cordão simples, pérgula, espaldeira e lira. Avaliaram-se as variáveis: massa e número médio de ramos; crescimento dos ramos; número de cachos; produção/planta; produtividade estimada/ha; análise química dos frutos; teor de sólidos solúveis totais ( ${ }^{\circ}$ Brix); acidez total titulável (meq...-1) e pH. Verificou-se que os sistemas espaldeira, lira e pérgula promoveram as maiores médias de massa e número de ramos em ambas variedades. Os sistemas de condução pérgula e cordão simples propiciaram uma certa precocidade em ambas cultivares. $\mathrm{O}$ porte descendente promovido pelo cordão simples induziu um menor crescimento de ramos. Os sistemas de condução foram semelhantes para a variável produção/planta para a cultivar 'Niagara Rosada' enquanto que para 'Folha de Figo' a lira foi superior. Os sistemas de condução lira e espaldeira apresentaram videiras com maiores produtividades para as cultivares estudadas. E as videiras conduzidas nos sistemas cordão simples e pérgula produzem frutos com melhor qualidade para ambas cultivares.
\end{abstract}

Termos para indexação: Uva, Sistema de condução, Produtividade e Qualidade, Vitis labrusca L.

\section{ABSTRACT}

The present work was developed in the Station of Viticulture and Enology of Caldas EPAMIG, Caldas, MG, and it aimed to evaluate the influence of the conduction system in the production and in the quality of the fruits of the vines 'Folha de Figo' and 'Niagara Rosada' during the crops 2003, 2004 and 2005. As you cultivate cup it was used Rosy Niagara and Leaf of Fig, grafted in the door-graft ' $420-\mathrm{TO}$ '. The experimental design was fully randomized, disposed in factorial scheme $4 \times 2$. The conduction systems adopted were: simple string, pergola, espaldeira and lira. The following variables were evaluated: mass and medium number of branches; shoot growth; shoot number; plant production; estimated yield; of total soluble solids levels ( ${ }^{\circ}$ Brix), total titrable acidity (meq. $\mathrm{L}^{-1}$ ), and $\mathrm{pH}$. It was verified that the systems espaldeira, lira and pergola promoted the highest mass averages and shoot number in both varieties. The conduction system Pergola and simple string triggered certain precocity in both varieties. The descending load promoted by the simple string induced a lower shoot growth. The conduction systems were similar for the variable production/plant for the cultivars 'Niagara Rosada,'while for 'Folha de Figo'the lira was higher. In the general, the systems of conduction lira and espaldeira showed vines with higher productivities for the analyzed cultivars. And the vines from the simple string and pergola systems produced fruits with better quality for both cultivars.

Index terms: Grape, Training System, Yield Quality, Vitis labrusca L.

(Recebido em 26 de abril de 2006 e aprovado em 1 de novembro de 2006)

\section{INTRODUÇÃo}

A videira é uma planta que se adapta a uma grande diversidade de arquiteturas não somente em seu dossel vegetativo mas também de suas partes perenes. A distribuição espacial do dossel vegetativo, do tronco e dos ramos, aliados a outras variáveis como altura do tronco, densidade de plantas e orientação das linhas de plantio constituem o sistema de condução da videira. Plantas conduzidas em um determinado ambiente permitem regular

\footnotetext{
${ }^{1}$ Parte da tese de Doutorado do primeiro autor apresentada à Universidade Federal de Lavras/UFLA - Cx. P. 3037 - $37200-000$ - Lavras, MG ${ }^{2}$ Doutor - Departamento de Agrigultura/DAG - Universidade Federal de Lavras/UFLA - Cx. P. 3037 - 37200-000 - Lavras, MG - paulonor@ufla.br ${ }^{3}$ Doutor, Pesquisador - Empresa Agropecuária de Minas Gerais/EPAMIG - Fazenda Experimental de Caldas/FECD - Cx. P. 33 - $37780-000$ - Caldas, MG murillo@epamigcaldas.gov.br

${ }^{4}$ Doutor, Professor Titular - Departamento de Agricultura/DAG - Universidade Federal de Lavras/UFLA - Cx. P. 3037 - $37200-000$ - Lavras, MG - nnagib@ufla.br ${ }^{5}$ Doutora, Professora Adjunto - Departamento de Biologia/DBI - Universidade Federal de Lavras/UFLA - Cx. P. 3037 - $37200-000$ - Lavras, MG amsoares@ufla.br

${ }^{6}$ Mestranda - Departamento de Ciências Exatas/DEX - Universidade Federal de Lavras/UFLA - Cx. P. 3037 - $37200-000$ - Lavras, MG vanessabf2004@yahoo.com.br
} 
melhor os fatores ambientais e as respostas fisiológicas para a obtenção do produto desejado, seja uva para o consumo in natura ou obtenção de matéria prima para elaboração de vinhos e outros derivados.

A escolha do sistema de condução mais adequado em viticultura deve levar em conta diversos aspectos tais como topografia, clima, destino da produção e disponibilidade de mecanização.

No sul de Minas Gerais, emprega-se tradicionalmente o sistema de condução em espaldeira, escolhido de forma empírica e sem o devido respaldo comparativo com outras formas de condução, que poderiam contribuir para o aumento, tanto da produtividade quanto da qualidade das uvas e ao mesmo tempo reduzir os custos de instalação do vinhedo. Como as videiras aí cultivadas são comuns e bem adaptadas ao clima local, torna-se importante avaliar o comportamento produtivo das mesmas em outros sistemas de condução.

Segundo Kliewer et al. (2000) para qualquer cultivar de uva, a variação sazonal na capacidade de frutificação de gemas deve-se a fatores climáticos, práticas culturais ou doenças. Entre os fatores climáticos mais estudados estão luz, temperatura, deficiência hídrica e fotoperíodo. Práticas culturais de maior influência na capacidade de frutificação das plantas incluem a poda e o uso de sistema de condução adequado.

Dessa maneira, o fator sistema de condução do vinhedo pode afetar significativamente o crescimento vegetativo da videira, a produtividade do vinhedo e a qualidade da uva e do vinho. Isso pode ocorrer em função do efeito do sistema de condução sobre a parte aérea e subterrânea da videira (MIELE \& MANDELLI, 2005).

Na região do sul de Minas Gerais as condições climáticas normalmente não são adequadas à época de maturação da uva, devido à alta precipitação e elevada nebulosidade, reduzindo assim a qualidade dos frutos e, por conseqüência, a do vinho (NOGUEIRA, 1984).

Conforme Toda et al. (1991), a radiação solar na região do cacho é mais importante durante a fase de maturação pelo seu efeito na composição do mosto, na acidez total, $\mathrm{pH}$ e antocianinas. Além disso folhas mais expostas ao sol promovem a diferenciação das gemas e favorecem o acúmulo de reservas nas bagas e a salificação dos ácidos orgânicos (RIDOMI \& MORETTI, 1996). Por outro lado dosséis vegetativos densos proporcionam menor produtividade da videira e menor qualidade do fruto (SMART, 1985).

Assim a escolha correta do sistema de condução no sul de Minas Gerais ganha grande importância para os que privilegiam uma melhor exposição da folha à luz a fim de atenuar os efeitos adversos dos fatores climáticos na área vinícola desta região (CARBONNEAU, 1991; REGINA et al., 1998). O objetivo deste trabalho foi avaliar a influência de diferentes sistemas de condução na produção e qualidade dos frutos das videiras 'Niagara Rosada' e 'Folha de Figo', nas condições climáticas da região sul-mineira.

\section{MATERIAL E MÉTODOS}

O trabalho foi desenvolvido na Estação de Viticultura e Enologia da Empresa de Pesquisa Agropecuária de Minas Gerais (EPAMIG), no município de Caldas, MG, durante as safras 2003, 2004 e 2005.

As cultivares copa pesquisadas foram a 'Folha de Figo', sinonímia regional da cultivar "Bordô" ou "Ives" e 'Niagara Rosada', enxertadas no porta - enxerto '420-A'.

$\mathrm{O}$ delineamento experimental foi inteiramente casualizado em fatorial $4 \times 2$, quatro sistemas de condução e duas cultivares, com quatro repetições de quatro plantas/ parcela, perfazendo um total de 128 plantas. A análise de variância foi realizada pelo teste $\mathrm{F}$ e o teste utilizado para comparação das médias foi o Scott-Knott a $5 \%$ de probabilidade para todas as variáveis. Tanto para a análise de variância quanto para os testes de média, utilizou-se o programa SISVAR (FERREIRA, 1999).

As características avaliadas nas safras 2003, 2004 e 2005 foram: a) massa em (g) e número médio de ramos; b) crescimento dos ramos em $(\mathrm{Cm})$ com medições semanais do crescimento de dois ramos/planta/subparcela; c) número total de cachos de cada subparcela; d) produção - pelo peso dos cachos de cada parcela em balança de campo, onde se estimou também a produtividade do vinhedo em t.ha $\left.{ }^{-1} ; \mathbf{e}\right)$ análise química dos frutos - iniciada a partir do estágio final de maturação ("pintor") até à ocasião da colheita - com avaliações semanais dos teores de sólidos solúveis totais ( ${ }^{\circ}$ Brix); da acidez total titulável em meq.L ${ }^{-1}$ e do pH.

\section{RESULTADOS E DISCUSSÃO}

\section{Massa e número de ramos por planta}

A massa e o número de ramos por planta avaliados por ocasião da poda de inverno, na safra 2005 e para os dados médios das três safras para 'Folha de Figo' e 'Niagara Rosada' foram influenciados pelo sistema de condução (Tabela 1). Para 'Folha de Figo', as maiores massas de ramos foram obtidas pelos sistemas espaldeira e lira com 2,19 e 2,38 kg. $\mathrm{pl}^{-1}$, respectivamente.

Em relação à média das três safras, para 'Folha de Figo', os melhores resultados para a massa e número de ramos foram obtidos nos sistemas pérgula, lira, e espaldeira e pérgula e espaldeira, respectivamente. Para 'Niagara Rosada', o sistema espaldeira apresentou maior número de ramos que os demais sistemas, totalizando 21 ramos/planta. 


\section{Curva de crescimento dos ramos principais}

Os resultados obtidos para a curva de crescimento dos ramos principais das cultivares 'Folha de Figo' e 'Niagara Rosada' durante as três safras avaliadas são apresentadas nas Figuras 1 e 2, onde se verifica que os sistemas espaldeira e lira proporcionaram desenvolvimento mais rápido dos ramos, nas duas cultivares em relação ao sistema cordão simples. O sistema pérgula permaneceu em posição intermediária. Esse resultado confirma as afirmativas de Carbonneau \& Zhang (1989) e Kliewer et al. (2000), em que a utilização de sistemas de condução, que promovem um porte descendente dos ramos, reduzem o vigor da planta pela redução da acrotonia.

Tabela 1 - Massa e número de ramos por planta das videiras 'Niagara Rosada'e 'Folha de Figo' sobre diferentes sistemas de condução. EPAMIG/ Caldas, 2005.

Dados Médios das safras 2003, 2004 e 2005

\begin{tabular}{l|cccc}
\hline \multirow{2}{*}{ Cultivares } & \multicolumn{2}{c}{ 'Folha de Figo' } & \multicolumn{2}{c}{ 'Niagara Rosada' } \\
\cline { 2 - 5 } Sist. Cond. & Massa de Ramos $(\mathrm{g})$ & $\mathrm{N}^{\circ}$ de Ramos & Massa de Ramos (g) & $\mathrm{N}^{\circ}$ de Ramos \\
\hline Cordão Simples & $1,17 \mathrm{~b}$ & $21 \mathrm{~b}$ & $1,41 \mathrm{a}$ & $16 \mathrm{~b}$ \\
Pérgula & $1,96 \mathrm{a}$ & $24 \mathrm{a}$ & $1,35 \mathrm{a}$ & $18 \mathrm{~b}$ \\
Espaldeira & $1,71 \mathrm{a}$ & $25 \mathrm{a}$ & $2,02 \mathrm{a}$ & $21 \mathrm{a}$ \\
Lira & $1,84 \mathrm{a}$ & $22 \mathrm{~b}$ & $1,71 \mathrm{a}$ & $18 \mathrm{~b}$ \\
\hline
\end{tabular}

* Médias seguidas pela mesma letra na coluna não diferem estatisticamente entre si pelo teste de Scott-Knott ao nível de 5\% de probabilidade.

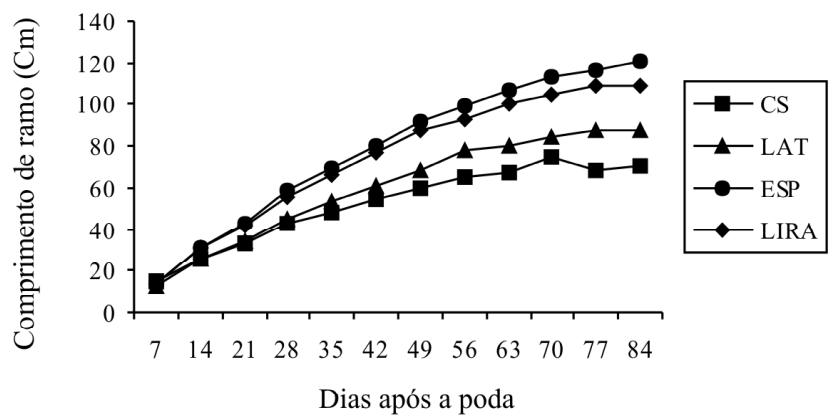

Figura 1 - Crescimento dos ramos da cultivar 'Folha de Figo' conduzida em diferentes sistemas de condução durante as safras 2003, 2004 e 2005 EPAMIG/ Caldas, 2005.

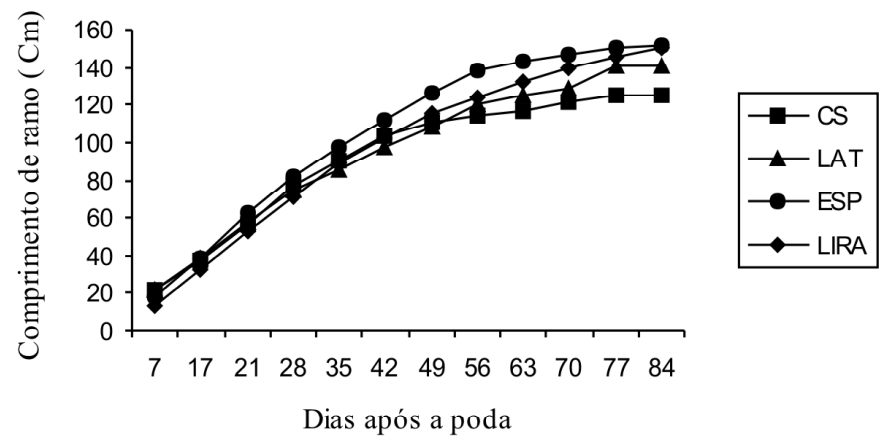

Figura 2 - Crescimento dos ramos da cultivar 'Niagara Rosada' conduzida em diferentes sistemas de condução durante as safras 2003, 2004 e 2005 EPAMIG/ Caldas, 2005. 


\section{Número de cachos, produção e produtividade estimada}

Os dados médios das safras 2003, 2004 e 2005 indicam que a condução em cordão simples mostrou-se superior aos demais apenas para a cultivar 'Niagara Rosada' com 30,42 cachos/planta-1 Orlando et al. (2003). Na região sul-mineira, constatou-se que, na cultivar 'Niagara Rosada', durante a safra 2001, a maior produção e número de cachos foram verificados nas plantas conduzidas no sistema espaldeira com 4,59 kg.pl-1 e 24 cachos.planta ${ }^{-1}$, respectivamente (Tabela 2 ).

Tabela 2 - Número de cachos por planta, produção /planta ${ }^{-1}$, e produtividade/ha estimada das cultivares 'Folha de Figo' e 'Niagara Rosada' em diferentes sistemas de condução. Dados médios das safras 2003, 2004 e 2005. EPAMIG, Caldas-MG, 2005.

\begin{tabular}{|c|c|c|}
\hline Cultivares & $\begin{array}{c}\text { 'Folha de } \\
\text { Figo' }\end{array}$ & $\begin{array}{l}\text { 'Niagara } \\
\text { Rosada' }\end{array}$ \\
\hline S.de Cond. & Dados Médios & Dados Médios \\
\hline \multicolumn{3}{|c|}{ Numero Médio de Cachos. Planta ${ }^{-1}$} \\
\hline Pérgula & $30,34 \mathrm{a}$ & $18,27 \mathrm{c}$ \\
\hline Cordão Simples & 46,15 a & 30,42 a \\
\hline Lira & $45,40 \mathrm{a}$ & $17,61 \mathrm{c}$ \\
\hline Espaldeira & 38,71 a & $24,48 \mathrm{~b}$ \\
\hline \multicolumn{3}{|c|}{ Produção Média $\left(\mathrm{Kg} \cdot \mathrm{pl}^{-1}\right)$} \\
\hline Pérgula & $4,22 \mathrm{~b}$ & 7,16 a \\
\hline Cordão Simples & $4,41 \mathrm{~b}$ & 7,14 a \\
\hline Lira & $5,91 \mathrm{a}$ & 7,08 a \\
\hline Espaldeira & $4,08 \mathrm{~b}$ & 6,82 a \\
\hline \multicolumn{3}{|c|}{ Produtividade Estimada $\left(\mathrm{Kg} \mathrm{ha}^{-1}\right)$} \\
\hline Pérgula & $8441,74 \mathrm{~b}$ & $14320,10 \mathrm{~b}$ \\
\hline Cordão Simples & $5031,20 \mathrm{c}$ & $8160,27 \mathrm{c}$ \\
\hline Lira & 16893,39 a & 20233,99 a \\
\hline Espaldeira & $10843,50 \mathrm{~b}$ & 18190,74 a \\
\hline
\end{tabular}

*Médias seguidas pela mesma letra na coluna não diferem estatisticamente entre si pelo teste de Scott-Knott ao nível de $5 \%$ de probabilidade.

Paulleto et al. (2001) verificaram produções médias menores que as encontradas neste trabalho, em plantas da cultivar 'Niagara Rosada', no município de Taubaté - SP, conduzidas em espaldeira em fase de formação com 2,69 $\mathrm{kg} /$ planta $^{-1}$.

A produção média, por planta, nas safras 2003, 2004 e 2005, da cultivar 'Folha de Figo', foi superior na condução em lira com 5,91 kg.pl ${ }^{-1}$, e para 'Niagara Rosada' não houve diferença significativa entre os sistemas de condução.

Para os resultados de produtividade estimada verificou-se que, para 'Folha de Figo', as maiores produtividades foram conseguidas nos sistemas lira, espaldeira e pérgula com 16893,39 kg.ha-1 $, 10843,50 \mathrm{~kg} \cdot \mathrm{ha}^{-1}$ e $8441,74 \mathrm{~kg} \cdot \mathrm{ha}^{-1}$, respectivamente. E a menor produção foi verificada no sistema de condução cordão simples com 5031,20 kg.ha- ${ }^{-1}$ em média nas três safras avaliadas, embora esse dado possa ter sido influenciado pela menor densidade de plantio, conferida por este sistema.

Maia et al. (1999) verificaram que plantas da cv. Isabel conduzidas em diversos sistemas de condução proporcionaram as seguintes produtividades: pérgula, com 47.042 kg.ha- ${ }^{-1}$, GDC, com 33.217 kg.ha- ${ }^{-1}$ e espaldeira com $17.450 \mathrm{~kg} \cdot \mathrm{ha}^{-1}$.

Para 'Niagara Rosada', as maiores produtividades foram verificadas nos sistemas de condução lira e espaldeira, na média das três safras com $(20.233,99$ e $18.190,74 \mathrm{~kg} \cdot \mathrm{ha}^{-1}$ ), respectivamente. Os menores valores de produtividade foram semelhantes aos obtidos para 'Folha de Figo', onde o sistema cordão simples conferiu $8.160,27 \mathrm{~kg} \cdot$ ha $^{-1}$, na média das três safras avaliadas.

Orlando et al. (2003), na região sul de Minas Gerais, verificaram também que as plantas conduzidas em espaldeira alcançaram produtividade de $10.632,40 \mathrm{~kg}^{-h^{-1}}$, sendo superior aos demais tratamentos, na safra 2001. Na safra 2002, a produtividade das plantas conduzidas em lira foi estatisticamente semelhante àquela das plantas conduzidas em espaldeira, alcançando média de $11.291,94 \mathrm{~kg} \cdot$ ha $^{-1}$, enquanto os sistemas cordão simples e pérgula produziram $6.670,00$ e $3.611,80 \mathrm{~kg} \cdot \mathrm{ha}^{-1}$, respectivamente. Enfim, a 'Niagara Rosada', se apresenta como ótima alternativa para a região devido ao menor custo com mão-de-obra; por não necessitar de raleio de bagas; apresentar menor suscetibilidade às doenças fúngicas; possuir boa aceitação no mercado interno, alcançando preços de $\mathrm{R} \$ 1,80 \mathrm{~kg}^{-1}$, podendo proporcionar bons rendimentos para o viticultor, sobretudo se conduzidas em espaldeira e lira.

\section{Acidez Total Titulável (ATT), Teor de Sólidos Solúveis (TSS) e pH}

Verifica-se na Tabela 3 e Figura 3, que a acidez total titulavel média de 135,56 meq. $\mathrm{L}^{-1}$ obtida nas safras 2003, 2004 e 2005 para a cultivar 'Folha de Figo', foi maior quando conduzida no sistema espaldeira, que também apresentou em média $9,90^{\circ}$ Brix, portanto o menor teor de sólidos solúveis totais (TSS). 
Tabela 3 - Teor de acidez total titulável, teor de sólidos solúveis e pH das videiras 'Folha de Figo' e 'Niagara Rosada' conduzidas sob diferentes sistemas de condução. EPAMIG, Caldas-MG, 2005.

\begin{tabular}{|c|c|c|c|}
\hline \multicolumn{4}{|c|}{ Dados Médios das safras 2003, 2004 e 2005} \\
\hline \multicolumn{4}{|c|}{ 'Folha de Figo' } \\
\hline Sistema de Condução & $\begin{array}{c}\text { Acidez Total Titulável } \\
\left(\mathrm{meg}, \mathrm{L}^{1}\right)\end{array}$ & $\begin{array}{c}\text { Teor de Sólidos Solúveis } \\
\text { ( }{ }^{\circ} \text { Brix) }\end{array}$ & $\mathrm{pH}$ \\
\hline Cordão Simples & 119,29 a & 10,29 a & $2,47 \mathrm{a}$ \\
\hline Pérgula & $119,41 \mathrm{a}$ & 10,19 a & $2,48 \mathrm{a}$ \\
\hline Lira & $124,78 \mathrm{a}$ & 10,19 a & $2,45 \mathrm{~b}$ \\
\hline Espaldeira & $135,56 \mathrm{~b}$ & $9,90 \mathrm{~b}$ & $2,46 \mathrm{~b}$ \\
\hline \multicolumn{4}{|c|}{ 'Niagara Rosada' } \\
\hline Sistema de Condução & $\begin{array}{c}\text { Acidez Total Titulável } \\
\left(\mathrm{meg}, \mathrm{L}^{1}\right)\end{array}$ & $\begin{array}{l}\text { Teor de Sólidos Solúveis } \\
\text { ( }{ }^{\circ} \text { Brix) }\end{array}$ & $\mathrm{pH}$ \\
\hline Cordão Simples & 81,63 a & $14,60 \mathrm{a}$ & $3,15 \mathrm{~b}$ \\
\hline Pérgula & 83,23 a & $14,09 \mathrm{~b}$ & 3,18 a \\
\hline Lira & $93,83 \mathrm{c}$ & $14,04 \mathrm{~b}$ & $3,09 \mathrm{c}$ \\
\hline Espaldeira & $89,48 \mathrm{~b}$ & $13,78 \mathrm{c}$ & $3,09 \mathrm{c}$ \\
\hline
\end{tabular}

*Médias seguidas pela mesma letra, na coluna, não diferem estatisticamente entre si pelo teste de Scott-Knott, ao nível de 5\% de probabilidade.

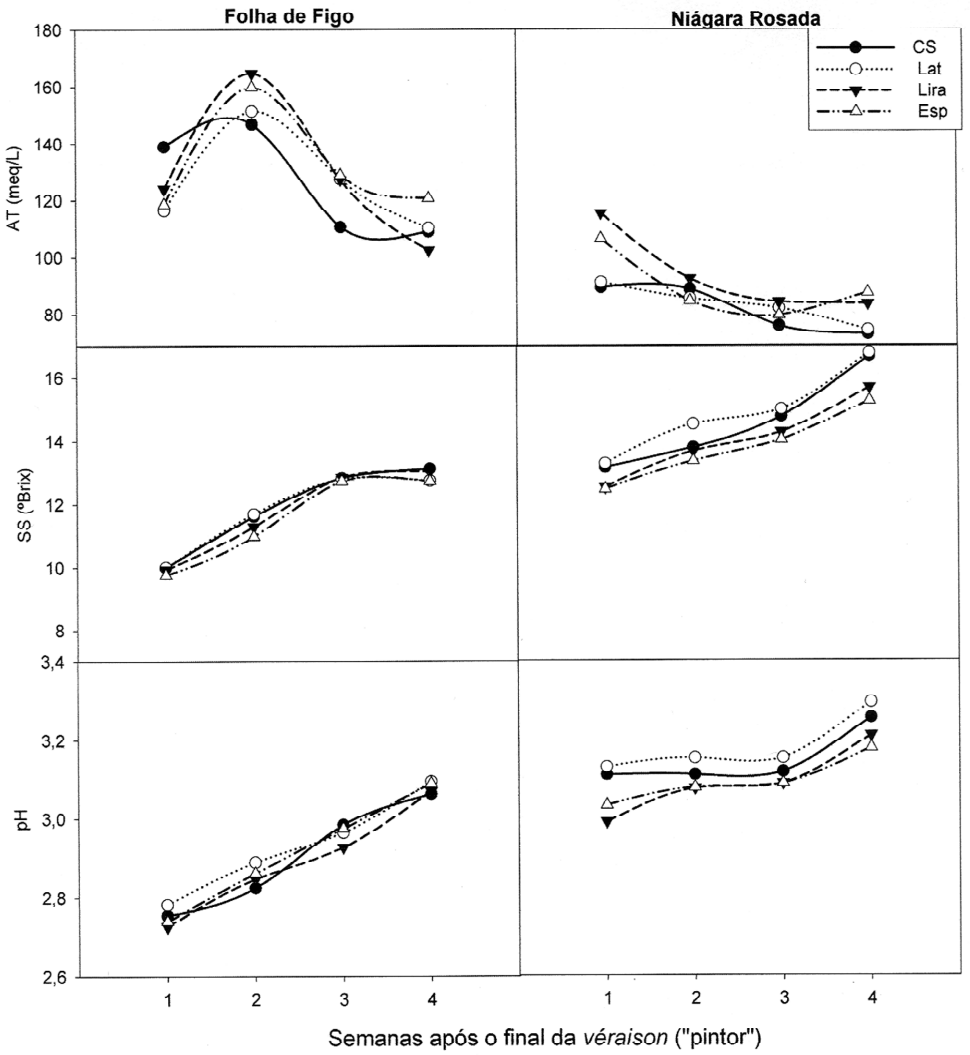

Figura 3 - Evolução média dos teores de acidez total titulável, sólidos solúveis e pH das videiras ‘Folha de Figo' e 'Niagara Rosada' conduzidas sob diferentes sistemas de condução, nas safras 2003, 2004 e 2005 EPAMIG, Caldas-MG, 2005. 
Nos demais sistemas de condução o teor médio foi de 10,22 ${ }^{\circ}$ Brix, também considerado baixo para elaboração de vinhos. Ainda para a cultivar 'Folha de Figo', verifica-se na Tabela 3 e Figura 3, que o pH variou de 2,45 a 2,48 sendo que os maiores valores ocorreram quando as videiras foram conduzidas em pérgula e cordão simples. Para 'Niagara Rosada' o maior teor de acidez total titulável foi verificado no sistema lira seguido da espaldeira, sendo que os demais sistemas apresentaram valores semelhantes entre si. Com relação ao teor de sólidos solúveis totais (TSS), pode-se observar que o valor mais expressivo foi conferido pelo cordão simples com $14,60^{\circ}$ Brix. Quanto ao $\mathrm{pH}$ médio observado nas amostras, o mais elevado foi conferido pelo sistema pérgula, seguido pelo cordão simples.

\section{CONCLUSÕES}

Os sistemas de condução lira , pérgula e espaldeira promoveram valores maiores de massa e número de ramos em ambas cultivares.

Os sistemas de condução lira e espaldeira apresentaram videiras com maiores produtividades para as cultivares estudadas.

Os sistemas cordão simples e pérgula produzem frutos com melhor qualidade para as cultivares estudadas.

No geral, os sistemas de condução lira e espaldeira foram os mais indicados para a região de Caldas- MG.

\section{REFERÊNCIAS BIBLIOGRÁFICAS}

CARBONNEAU, A. Etude écophysiologique des principaux systèmes de conduite interêt qualitatif et économique des vignes en Lyre: premières indications de leur comportement en situation de vigueur élevée. In: CONGRESSO BRASILEIRO DE VITICULTURA E ENOlOGIA, 6., 1990, Bento Gonçalves. Anais... Bento Gonçalves: Embrapa/CNPUV/ABTE/OIV, 1991. p. 21- 34 .

CARBONNEAU, A.; ZHANG, D. P. Influence of winter pruning methods on grapevine physiology: consequences for mechanical pruning and justifications of the principle of the: "alternated crenel": originality of sap flow measurements in the trunk. In: INTERNATINAL SEMINAR ON MECHANICAL PRUNING OF VINEYARDS, 2., 1988, Treviso. Rivista di Ingegne, Treviso, v. 9, p. 121-132, 1989.

FERREIRA, F. D. SISVAR: sistema de análise de variância para dados balanceados. Lavras: DCE/UFLA, 1999. 18 p. Programa em disquete.
KLIEWER, W. M.; WOLPET, J. A.; BENZ, M. Trellis and vine spacing effects on growth, canopy microclimate, yield and fruit composition of Cabernet Sauvignon. Acta Horticulturae, Wageningen, v. 526, p. 21-31, 2000.

MAIA, J. D. G.; CAMARGO, U. A.; NATCHIGAL, J. C. Avaliação de cultivares de uvas americanas em Jales, noroeste paulista, período 1996/97/98. In: CONGRESSO BRASILEIRO DE VITICULTURA E ENOLOGIA, 10., 1999, Bento Gonçalves. Resumos... Bento Gonçalves: Embrapa Uva e Vinho, 1999. p. 157.

MIELE, A.; MANDELLI, F. Sistemas de condução da videira. Brasília, DF: Embrapa Uva e Vinho, 2005.

NOGUEIRA, D. J. P. Porta-enxertos de videira. Informe Agropecuário, Belo Horizonte, v. 10, n. 117, p. 22-24, set. 1984.

ORLANDO, T. G. S.; REGINA, M. A.; SOARES, A. M.; CHALFUN, N. N. J.; SOUZA, C. M.; FREITAS, G. F.; TOYOTA, M. Caracterização agronômica de cultivares de videira (vitis labrusca 1.) em diferentes sistemas de condução. Ciência e Agrotecnologia, Lavras, p. 1460-1469, dez. 2003. Edição especial.

PAULETTO, D.; MACHADO FILHO, F. de A.; KLUGE, R. A.; SOUZA FILHO, J. A. Produção e vigor da videira 'Niagara Rosada' relacionados com o porta-enxerto. Pesquisa Agropecuária Brasileira, Brasília, v. 36, n. 1, p. 115-121, jan. 2001

REGINA, M. de A.; PEREIRA, A. F.; ALVARENGA, A. A.; ANTUNES, L. E. C.; ABRAHÃO, E. Sistema de condução da videira. Informe Agropecuário, Belo Horizonte, v. 19, n. 194, p. 5-8, set. 1998.

RIDOMI, A.; MORETTI, G. Comportamento della cv. Cabernet Franc (Vitis vinifera L.) allevata in cinque combinazioni d'innesto e con due sesti d'impianto. Vignevini, Bologna, v. 23, n. 7/8, p. 3-8, 1996.

SMART, R. E. Principles of grapevine canopy microclimate manipulation with implications for yield and quality: a review. American Journal of Enology and Viticulture, Davis, v. 36, n. 3, p. 230-239, 1985.

TODA, F. M. de; SANCHA, J. C.; LLOP, E. Estudio comparado del microclima luminoso en los sistemas de conducción en vaso y espaldera en Rioja. Rivista di Viticoltura e di Enologia, Treviso, v. 44, n. 4, p. 149-156, 1991. 